\title{
EVIDENCIAS DEL ORIGEN DE NASUA Y PROCYON (PROCYONIDAE: CARNIVORA) EN AMÉRICA DEL SUR
}

\author{
DAMIÁN RUIZ-RAMONI \\ Depto. Paleontología, Instituto de Geología, Universidad Nacional Autónoma de México, Cd. Universitaria, 04510, Ciudad \\ de México, CDMX, México.drramoni@gmail.com

\section{ASCANIO RINCÓN} \\ Laboratorio de Paleontología, Centro de Ecología, Instituto Venezolano de Investigaciones Científicas, Km 11 de la \\ Carretera Panamericana, Estado Miranda. Aptdo. 21.827, Cod., 1020-A, Caracas, Venezuela. paleosur1974@gmail.com

\section{MARISOL MONTELLANO-BALLESTEROS} \\ Depto. Paleontología, Instituto de Geología, Universidad Nacional Autónoma de México, Cd. Universitaria, 04510, Ciudad \\ de México, CDMX, México.marmont@unam.mx
}

\begin{abstract}
Evidence of the origin of Nasua and Procyon (Procyonidae: Carnivora) in South America. In this work, fossil material of Nasua and Procyon from the Marplatan of El Breal of Orocual, Venezuela, is reported as the oldest record of these procyonids in South America. This gives evidence of an earlier origin of these genera in the continent than was previously thought.
\end{abstract}

Keywords: carnivorous, tar pit, El Breal de Orocual, Great American Biotic Interchange, Venezuela.

RESUMO - Neste trabalho, o material fóssil de Nasua e Procyon do Marplatense de El Breal de Orocual, Venezuela, é relatado como o registro mais antigo de procionídeos na América do Sul. Isso evidencia uma origem anterior desses gêneros no continente em relação ao que se pensava anteriormente.

Palavras-chave: carnívoro, poço de alcatrão, El Breal de Orocual, Grande Intercâmbio Biótico das Américas, Venezuela.

\section{INTRODUCCIÓN}

En América del Sur, los prociónidos (familia Procyonidae Gray, 1825) son un grupo de carnívoros muy diversos, con una historia biogeográfica y cronológica muy dinámica (Soibelzon \& Prevosti, 2012). Actualmente están representados en el continente por cinco géneros (Bassaricyon, Nasua, Nasuella, Potos y Procyon), existiendo además dos géneros extintos (Chapadmalania y Cyonasua) (Prevosti \& Soibelzon, 2012; Soibelzon \& Prevosti, 2012).

Los prociónidos aparecieron por primera vez en los estratos del Eoceno superior-Oligoceno superior del occidente de Europa (Wolsan \& Lange-Badré, 1996), y están registrados en América del Sur desde el Mioceno superior (Soibelzon \& Prevosti, 2012).

El primer grupo de prociónidos que ingresó a América del Sur estaba compuesto por miembros de los géneros Cyonasua y Chapalmalania, los cuales están registrados por primera vez para el Huayqueriense-Montehermosense (9,0-4,0 Ma.), y son considerados como parte de la fauna predecesora al Gran Intercambio Biótico de Las Américas
(GABI, por sus iniciales en inglés) (Simpson, 1950; Patterson \& Pascual, 1968; Woodburne, 2010; Soibelzon \& Prevosti, 2012; Prevosti et al., 2013; Forasiepi et al., 2014). De los géneros actuales, solamente Nasua y Procyon tienen registro fósil en el continente y presentan una ocurrencia reportada desde el Lujanense (0,009-0,126 Ma.) (Soibelzon et al., 2010; Rodríguez et al., 2013; Ramírez et al., 2015).

Existen dos hipótesis sobre el origen de Nasua y Procyon en América del Sur. Koepfli et al. (2007) sugirieron que Procyon y Nasua probablemente formaron parte del grupo original de prociónidos pre-GABI, e ingresaron al continente junto con Cyonasua, basado en que los tiempos de divergencia (estimados por vía de reloj molecular) entre las especies norteamericanas $(P$. lotor y $N$. narica) y suramericanas $(P$. cancrivorus y $N$. nasua) están ubicados sobre el Mioceno. Por otro lado, está la hipótesis de un ingreso más tardío (Simpson, 1950; Patterson \& Pascual, 1968; Woodburne, 2010; Soibelzon \& Prevosti, 2012; Prevosti et al., 2013; Rodríguez et al. 2013; Forasiepi et al., 2014). Sobre esta idea, Prevosti y Soibelzon (2012) señalan que estos géneros formaron parte de un pulso migratorio masivo durante el 
Lujanense, lo que ubicaría a estos mamíferos como uno de los últimos inmigrantes en el contexto del GABI. Ciertamente estos autores señalan que el registro fósil es limitado y que estos géneros pudieron haber tenido un ingreso más temprano, cercano al límite Ensenadense-Bonaerense (0,4 Ma.).

El nuevo descubrimiento de restos fósiles asignados a los géneros Nasua y Procyon para el Marplatense de El Breal de Orocual en Venezuela, refuta la hipótesis de un ingreso tardío a América del Sur.

\section{ÁREA DE ESTUDIO}

El Breal de Orocual ORS-16 está ubicado en los llanos orientales de Venezuela, al noroeste de la ciudad de Maturín en el Estado Monagas (Figura 1). El yacimiento se formó por la emanación natural de hidrocarburo sobre estratos de origen fluvio-deltaicos relacionados con el Paleorinoco en el Pleistoceno medio, y asociados a la Formación Mesa de edad Pleistoceno y Formación Las Piedras de edad Plioceno (Gonzáles et al., 1980; Carbón et al., 1992). Actualmente el yacimiento consiste en un depósito de asfalto o brea con elementos fósiles para el cual Rincón et al. (2009; 2011) sugieren una edad entre el Plioceno tardío y el Pleistoceno temprano basándose en los biocrones de Chapalmatherium sp., Pachyarmatherium leiseyi y Smilodon gracilis, lo que incluiría a esta localidad probablemente en el Marplatense superior (SALMA, siguiendo a Croft, 2016). La acumulación masiva de elementos esqueléticos sugiere que en el pasado este pozo pudo actuar como una trampa hiperacumuladora de restos de organismos, en especial mamíferos, que fueron quedando atrapados en la brea durante el tiempo en que el flujo de hidrocarburo se mantuvo activo. Hasta la fecha, se han reportado 27 mamíferos (que con los nuevos reportes en este trabajo aumenta a 29 taxones) (Rincón et al., 2007, 2009,2011; Ruiz-Ramoni, 2016; Ruiz-Ramoni et al., 2017).

\section{MATERIAL Y MÉTODOS}

Los elementos fósiles descritos en este trabajo están depositados en la Colección de Paleontología de Orocual (OR-) en el Instituto Venezolano de Investigaciones Científicas (IVIC), Estado Miranda, Venezuela. Se realizaron comparaciones morfométricas y descriptivas con material actual de Bassaricyon beddardi $(\mathrm{n}=6)$, Nasuella meridensis $(\mathrm{n}=2)$, Nasua nasua $(\mathrm{n}=4)$ y Procyon cancrivorus $(\mathrm{n}=$ 5) de la colección del Museo de la Estación Biológica de Rancho Grande (MEBRG) y N. narica $(\mathrm{n}=4)$ y P. lotor $(\mathrm{n}=5)$ del Natural History Museum Los Angeles County (LAM). Las medidas fueron tomadas con un vernier (Trupper, $\pm 0,1 \mathrm{~mm}$, máx. $150 \mathrm{~mm}$ ). Adicionalmente se consideraron los valores morfométricos reportados por Wrigth \& Lundelius Jr. (1963) para P. lotor, Oelrich (1953) para $P$. rexroandensis (recientemente Gilmore, 2013 ha sugerido que $P$. rexroandensis es sinónimo de $P$. lotor, sin embargo, en este trabajo se mantiene aún separadas), Baskin (1982) para Arctonasua (A. floridana, A. gracilis, A. minima y A. eurybate), Linares (1981) y Forasiepi et al. (2014) para

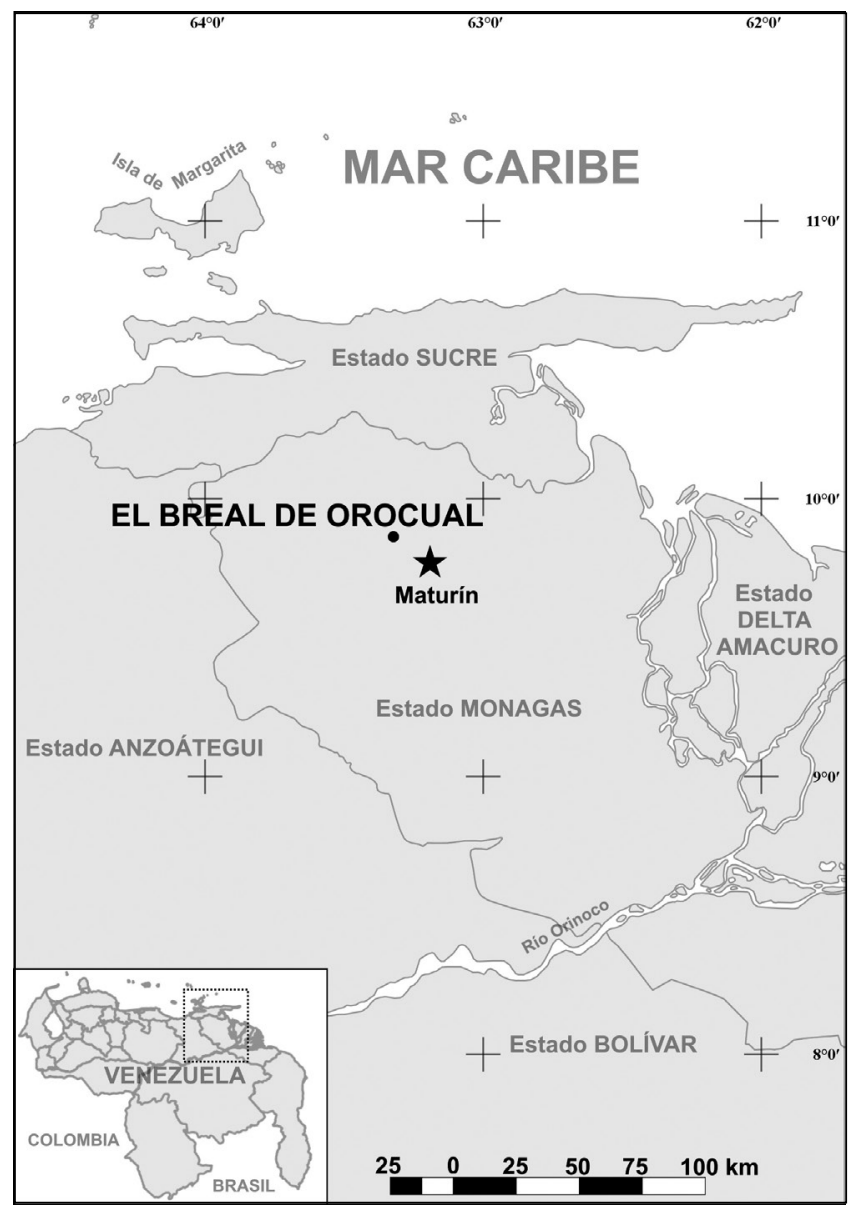

Figura 1. Ubicación del pozo de asfalto El Breal de Orocual en Venezuela. Estados Anzoátegui y Monagas forman parte de los llanos orientales venezolano.

Figure 1. Location of the tar pit El Breal de Orocual in Venezuela. Anzoátegui and Monagas states are part of the Venezuelan eastern plains.

Cyonasua (C. pascuali, C. lutaria, C. clausa y C. groeberi), y Linares (1981) para Protoprocyon savagei y Rhapsodus tedfordi (ver Apéndice 1 para la lista de ejemplares revisados).

\section{PALEONTOLOGÍA SISTEMÁTICA}

Orden CARNIVORA Bowdich, 1821

Familia PROCYONIDAE Gray, 1825

Subfamilia PROCYONINAE Gray, 1925

Tribu PROCYONINI Gray, 1825

Nasua Storr, 1780

Nasua nasua (Linnaeus, 1766)

(Figuras 2A-B)

Material referido. OR-6447: M1 izquierdo; OR-6051: m2 izquierdo. Medidas en Tabla 1.

Descripción y comparación. El M1 (OR-6447) es un diente cuadrangular en vista oclusal (Figura 2A), como en Nasua, 


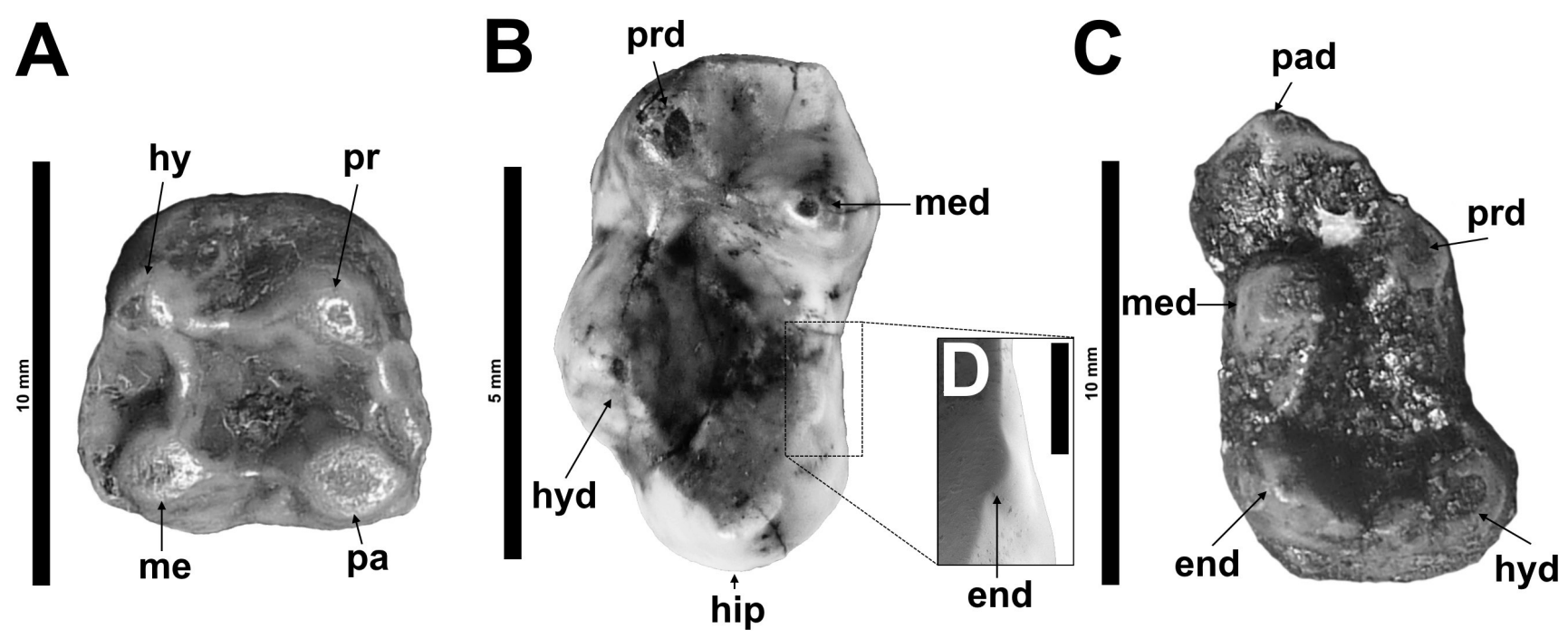

Figura 2. Nasua nasua (A-B) y Nasua sp. cf. N. nasua (C). A, M1 izquierdo (OR-6447) en vista oclusal; B, m2 izquierdo (OR-6051) en vista oclusal. C, m1 derecho (OR-3563) en vista oclusal. D, detalle del entocónido de OR-6051 (fotografía tomada con el Microscopio Electrónico de Barrido, QUANTA 250 FEI, de la Unidad de Microscopía Electrónica del Centro de Química-IVIC); Abreviaturas: end, entocónido; hip, hipoconúlido; hy, hipocono; hyd, hipocónido; me, metacono; med, metacónido; pa, paracono; pad, paracónido; pr, protocono; prd, protocónido. Escalas: A, C=10 mm; $\mathrm{B}=5 \mathrm{~mm} ; \mathrm{D}=1 \mathrm{~mm}$.

Figure 2. Nasua nasua (A-B) and Nasua sp. cf. N. nasua (C). A, left M1 (OR-6447) in occlusal view. B, left m2 (OR-6051) in occlusal view. C, right m1 (OR3563 ) in occlusal view. D, entoconid detail of OR-6051 (picture taken with Scanning Electron Microscope, QUANTA 250, FEI, of the Unidad de Microscopía Electrónica del Centro de Química-IVIC); Abbreviations: end, entoconid; hip, hypoconulid; h, hypocone; hyd, hypoconid; me, metacone; med, metaconid; pa, paracone; pad, paraconid; pr, protocone; prd, protoconid. Scale bars: $\mathrm{A}, \mathrm{C}=10 \mathrm{~mm} ; \mathrm{B}=5 \mathrm{~mm} ; \mathrm{D}=1 \mathrm{~mm}$.

Arctonasua y Procyon. El cíngulo lingual o interno está más desarrollado que en los miembros de la tribu Potosini (Bassaricynoides, Parapotos y Potos). Las cúspides son pequeñas y bien diferenciadas entre ellas. Están conectadas por medio de crestas, como ocurre en Nasua, mientras que en Procyon y Artonasua estas cúspides están más desarrolladas y no presentan crestas. Las cúspides están alineadas una con respecto a la otra en ejes perpendiculares, aunque en el caso del protocono se aprecia un ligero desplazamiento distal con respecto al paracono. Presenta un cíngulo distal bien diferenciado, como se aprecia en Nasua. Al igual que en $N$. nasua, el cíngulo lingual no se retrae en la corona provocando una marcada diferenciación del hipocono (como ocurre en $N$. narica). El hipocono es la cúspide más reducida. El metaconúlido está presente y ubicado mesialmente al protocono. El m2 (OR-6051) es alargado en el eje mesial/ distal, como en Bassariscus, Nasuella y Nasua. Las cúspides son altas y agudas, a diferencia de algunos Procyon (donde son ligeramente más bajas y globosas). El paracónido está ausente. El contacto con el $\mathrm{m} 1$ ocurre de manera sagital (en Bassariscus ocurre ligeramente lateral al plano sagital, aunque esto puede ser producto de variación intraespecífica). En el trigónido, las cúspides están más desarrolladas que en Nasuella. El protocónido y el metacónido están adyacentes (en Bassariscus estas cúspides están separadas) y alineados. El talónido es más alargado que el trigónido ( $64 \%$ del largo total). El entocónido está fusionado al hipoconúlido (ver Figura 2D) como ocurre en $N$. nasua (en $N$. narica esta cúspide está claramente diferenciada). El hipoconúlido es robusto y está desplazado distalmente al hipocono (en Nasuella la proyección distal del hipoconúlido es menor) y está igual de desarrollado que el metacónido (como en $N$. nasua).

Observaciones. OR-6447 y OR-6051 pertenecen a un prociónido con una morfología compatible al género Nasua. Decker (1991) hace una distinción entre las especies de este género y señala que $N$. nasua presenta un entocónido en el $\mathrm{m} 2$ y al contrario de $N$. narica. Posteriormente Gompper $\&$ Decker (1998) hacen una mayor revisión y señalan que en $N$. nasua el $\mathrm{m} 2$ tiene el entocónido ausente. Por otro lado, Gompper (1995) señala que $N$. narica se distingue el entocónido del resto de las cúspides. Bajo este contexto, en este trabajo se reconsidera la interpretación de esta cúspide en estas especies. Ambas especies presentan un entocónido, como señala Ahrens (2012), pero en N. narica este entocónido está reducido, aunque diferenciado del resto de las cúspides. Por su lado, en $N$. nasua el entocónido si está presente, pero fusionado al hipoconúlido (como se aprecia en la Figura 2D y se diagrama en la Figura 3). La asignación taxonómica de estos elementos queda confirmada por el desarrollo del cíngulo lingual en el M1, ya que en $N$. narica esta estructura está menos desarrollada.

\section{Nasua sp. cf. N. nasua (Linnaeus, 1766)}

(Figura 2C)

Material referido. OR-3563: $\mathrm{m} 1$ derecho; OR-2453: $\mathrm{m} 1$ derecho. Medidas en Tabla 1.

Descripción y comparación. El m1 (OR-3563) es alargado en el eje mesial/distal (Figura 2C) y no tan robusto como en Procyon. El trigónido está conformado por tres cúspides 
bien diferenciadas (paracónido, metacónido y protocónido) y aguzadas. El paracónido está ubicado mesialmente al protocónido y metacónido y es una cúspide ligeramente ensanchada lateralmente, como en Nasua. El paracónido está más desarrollado y diferenciado que en Cyonasua. El paracónido y metacónido están separados, como ocurre en $N$. nasua (en $N$. narica estas cúspides están ligeramente más unidas) y Rhapsodus tedfordi (contrario a algunos miembros del género Procyon, quienes presentan esta estructura unida al metacónido; ej. $P$. cancrivorus).

Observaciones. OR-3563 y OR-2453 corresponden a dos $\mathrm{m} 1$ morfológicamente similar Nasua nasua. Sin embargo, son un 49,33\% más grande que los miembros actuales (Figura 4), por lo cual no se asigna de manera absoluta a esta especie.

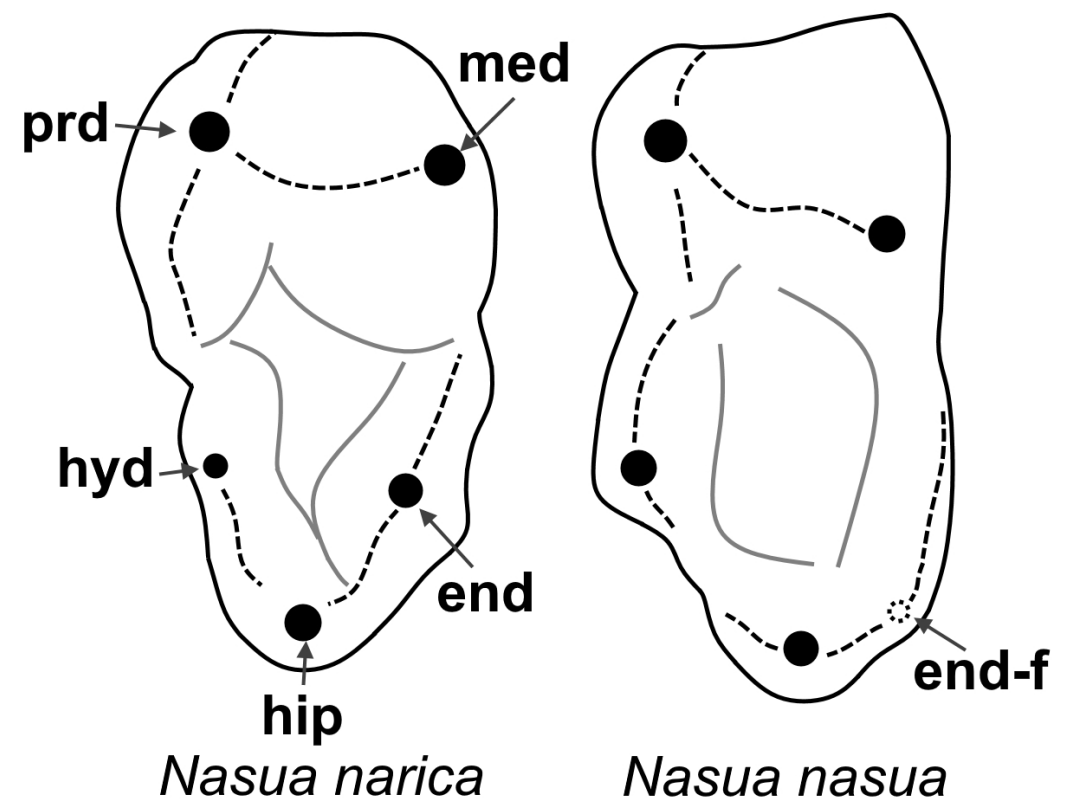

Figura 3. Esquema de la morfología oclusal del $\mathrm{m} 2$ de Nasua narica y $N$. nasua. Observe como en $N$. narica el entocónido está bien diferenciado y en $N$. nasua está fusionado al hipoconúlido. Abreviatura: end-f, entocónido fusionado al hipoconúlido.

Figure 3. Scheme of the occlusal morphology of the Nasua narica and N. nasua m2. Note how in N. narica the entoconid is well differentiated and in N. nasua is fused to the hypoconulid. Abbreviation: end-f, entoconid fused to the hypoconulid.

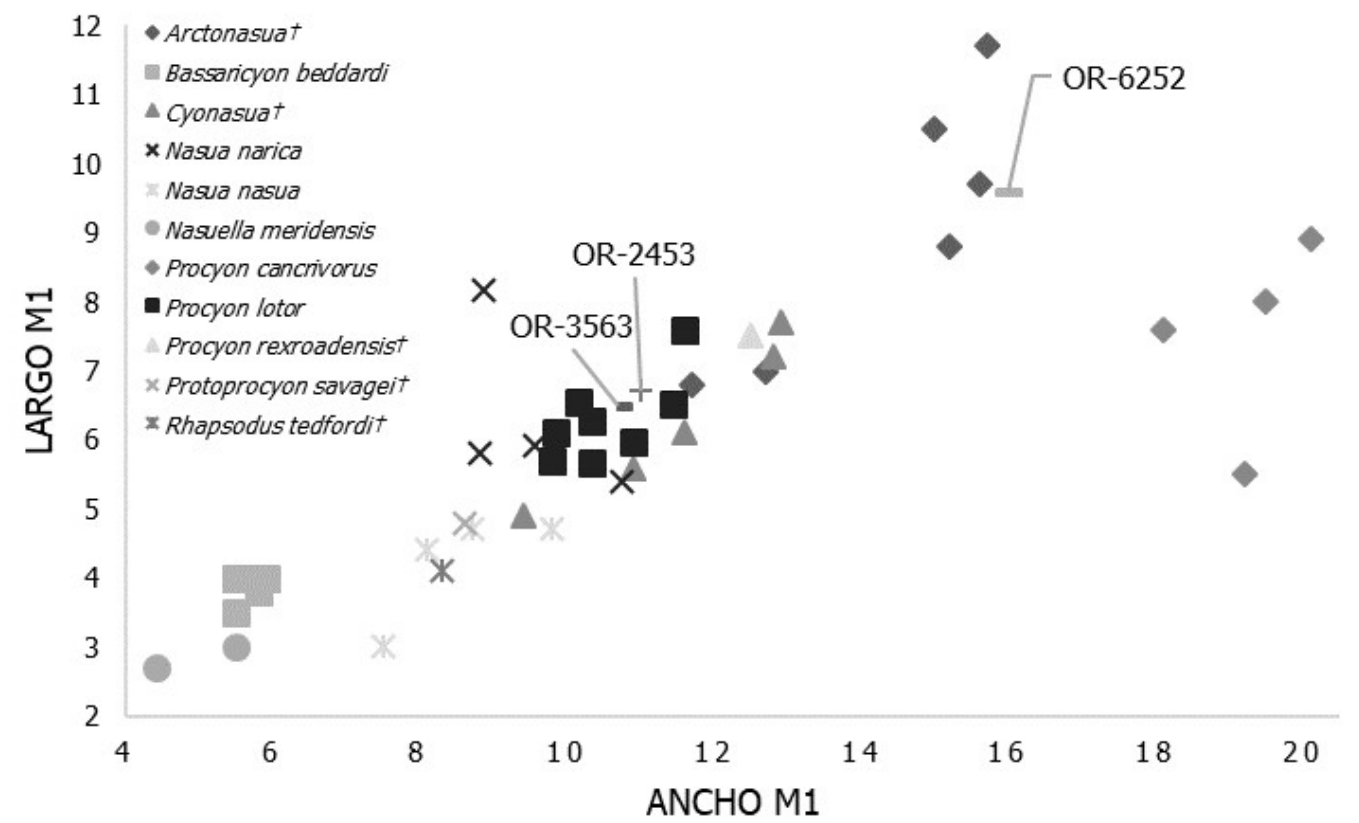

Figura 4. Relación del largo y ancho de los m1 OR-3563, OR-2453 (Nasua sp. cf. N. nasua) y OR-6252 (Procyon sp.) con respecto a algunos prociónidos (ver Material y Métodos).

Figure 4. Relationship of length and width of the m1 OR-3563, OR-2453 (Nasua sp. cf. N. nasua), and OR-6252 (Procyon sp.) with respect to some procyonids (see Material y Métodos). 
Procyon Storr, 1780

\section{Procyon sp.}

(Figura 5)

Material referido. OR-6252: corona de $\mathrm{m} 1$ derecho sin raíz (asociado con OR-6254); OR-6254: corona de m2 derecho sin raíz; OR-1137: M1 izquierdo sin raíces; OR-6253: M1 fragmentado con un elevado grado de desgaste (cámara pulpar expuesta); OR-702: I3 derecho; OR-2451: P2 derecho; OR704: P? izquierdo; OR-6239: p2 izquierdo sin raíz anterior. Medidas en Tabla 1.

Descripción y comparación. El I3 (OR-702) es caniniforme (Figura 5A) como ocurre en la mayoría de los prociónidos. La longitud de la corona es similar a la longitud de la raíz. Tiene una cúspide accesoria distal muy reducida (como en
Procyon, a diferencia de Nasua, Nasuella y Bassaricyon, donde esta cúspide está ausente). El P2 (OR-2451) tiene la cúspide principal cónica, ligeramente comprimida en el eje lingual/labial (menos que en Nasua o el cánido Urocyon). Tiene una cúspide accesoria posterior muy reducida, como se observa en Procyon. Presenta dos raíces que forman entre ellas un ángulo mayor que los premolares inferiores (Figura 5B). El M1 es cuadrangular (Figura 5C), a diferencia de Potos y Cyonasua. Tiene el parastilo muy reducido. El hipocono está presente y es la cúspide más pequeña, aunque está más desarrollado que en otros miembros del género; está desplazado labialmente, como se observa en Arctonasua floridana y el espécimen USNM 2634 de $P$. lotor, aunque no tanto como en $P$. rexroadensis (ver observaciones). El metaconúlido es prominente y está ubicado distalmente al protocono, como en Procyon. El metacono está conectado al

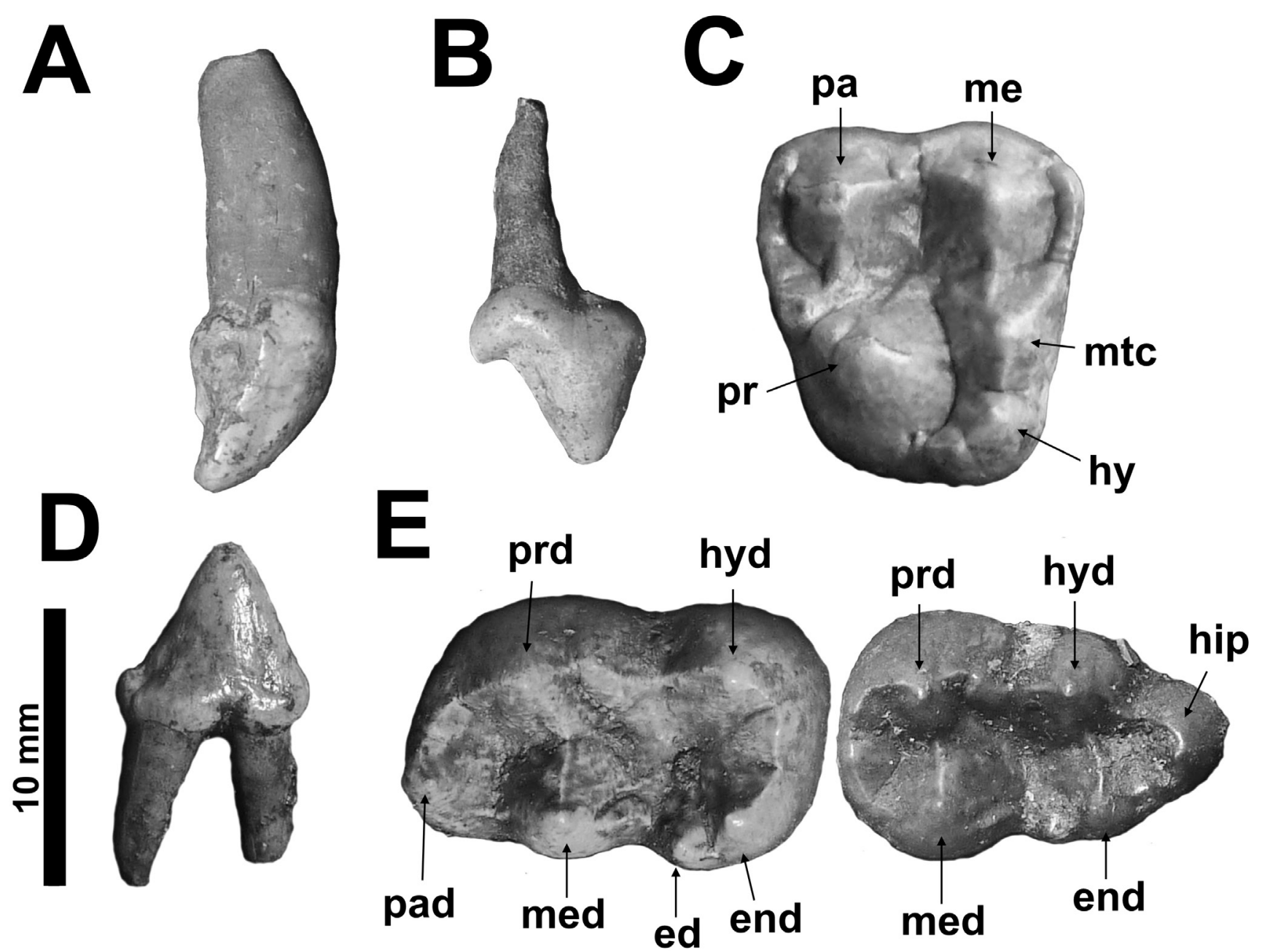

Figura 5. Procyon sp. A, I3 derecho (OR-702) en vista bucal. B, P2 derecho (OR-2451) en vista bucal. C, M1 izquierdo (OR-1137) en vista oclusal. D, p2 izquierdo (OR-6239) en vista bucal. E, m1 (OR-6252) y m2 (OR-6254) derecho en vista oclusal. Abreviaturas: el, entoconúlido; end, entocónido; hip, hipoconúlido; hy, hipocono; hyd, hipocónido; me, metacono; med, metacónido; mtc, metaconúlido; pa, paracono; pad, paracónido; pr, protocono; prd, protocónido. Escala $=10 \mathrm{~mm}$.

Figure 5. Procyon sp. A, right I3 (OR-702) in buccal view. B, right $\mathrm{P} 2$ (OR-2451) in buccal view. C, left M1 (OR-1137) in occlusal view. D, left p2 (OR-6239) in buccal view. E, right $\mathrm{m} 1$ (OR-6252) and m2 (OR-6254) in occlusal view. Abbreviations: el, entoconulid; end, entoconid; hip, hipoconulid; hy, hypocone; hyd, hypoconid; me, metacone; med, metaconid; mtc, metaconulid; pa, paracone; pad, paraconid; pr, protocone; prd, protoconid. Scale bar = 10 mm. 
metaconúlido por una cresta, aunque no tan prominente como en Nasua. El p2 (OR-6239) es cónico y menos alargado en el eje mesial/distal que Nasua. Presenta una cúspide accesoria distal en forma de gancho (Figura 5D), observada en Procyon. Posee dos raíces que forman un ángulo menor entre ellas que el formado por los premolares superiores. El m1 (OR-6252) es alargado en el eje mesial/distal (Figura 5E). Las cúspides del trigónido están bien diferenciadas y dispuestas en triangulo rectángulo, donde el paracónido está ligeramente desplazado distalmente, el metacónido y el protocónido están dispuestos paralelamente. El paracónido no está unido al metacónido, como en Rhapsodus tedfordi (a diferencia de P. cancrivorus, $P$. rexroadensis y $A$. floridana; en $P$. lotor estas cúspides están más separadas, pero no tanto como en OR-6252). El paracónido tiene una cúspide secundaría muy reducida en la región lingual de la paracrístida (menos desarrollada que en $P$. cancrivorus y $P$. lotor). Hay una cúspide muy reducida en la base distal del protocónido, que pudiera ser el mesocónido (en algunos ejemplares de $P$. cancrivorus se ha observado esta estructura más desarrollada que en OR-6252). El entocónido está presente y más desarrollado que en $P$. rexroandensis. Hay un entoconúlido en la base del entocónido, como en $P$. lotor y P. rexroandensis. El $\mathrm{m} 2$ es ligeramente más corto que el $\mathrm{m} 1$ (Tabla 1). Las cúspides están más definidas que en $P$. cancrivorus. El trigónido está conformado por el protocónido y metacónido (Figura 5E). El hipocónido está tan desarrollado como el protocónido. El entocónido está presente y bien diferenciado del hipoconúlido (a diferencia de Nasua). El hipoconúlido está proyectado distalmente, como en $P$. lotor y $P$. cancrivorus (en $P$. rexroandensis esta cúspide no se diferencia tanto del resto de la corona).

Observaciones. El material descrito en esta sección presenta una morfología similar con Procyon, pero a diferencia de los miembros reconocidos, es de mayor talla (Figura 4). Algunos caracteres en este ejemplar no han sido apreciados en otros miembros del género (ej. $\mathrm{m} 1$ con cúspides muy diferenciadas y separadas entre ellas, cúspide secundaria al lado del paracónido muy reducida), sin embargo, el desplazamiento lingual del hipocono en el M1 ha sido observado en los ejemplares UM 37131 de $P$. rexroadensis (Gilmore, 2013) y USNM 2634 de P. lotor (Gidley, 1908). Gilmore (2013) considera que el desplazamiento del hipocono de UM 37131 (Blanquense, NALMA) no es tan fuerte como en algunos ejemplares de $P$. lotor. Una condición similar pudiera ocurrir con el ejemplar USNM 2634 que fue clasificado como $P$. simus del Pleistoceno de California, el cual debido sus semejanzas morfológicas con material de $P$. lotor fue sinonimizada con esta especie (Wrigth \& Lundelius Jr., 1963). Debido a una combinación de caracteres entre distintos miembros del género Procyon no se descarta la posibilidad de un nuevo taxa. Sin embargo, algunos autores han señalado que estos prociónidos presentan una considerable variabilidad morfológica (Rodríguez et al., 2016) por lo cual es necesario mayor cantidad de material para resolver la taxonomía de este género en El Breal de Orocual. Por otro lado, Baskin (2004) señala que el género Procyon tiene un parastilo en el M1 alargado. A pesar de esto, el material referido aquí no lo
Tabla 1. Medidas dentales (en mm) de los prociónidos del Marplatense de El Breal de Orocual.

Table 1. Dental measurements (in mm) of the procyonids from the Marplatan of El Breal of Orocual.

\begin{tabular}{lcc}
\hline & $\begin{array}{c}\text { Largo } \\
\text { (mesiodistal) }\end{array}$ & $\begin{array}{c}\text { Ancho máximo } \\
\text { (bucolingual) }\end{array}$ \\
\hline Nasua nasua & $8,2^{*}$ & 7,9 \\
OR-6447 (M1) & 8,4 & 5,1 \\
OR-6051 (m2) & & \\
Nasua cf. N. nasua & 10,2 & 6,5 \\
OR-3563 (m1) & 10,5 & 6,7 \\
OR-2453 (m1) & & \\
Procyon sp. & 5,0 & 3,9 \\
OR-702 (I3) & 6,9 & 4,9 \\
OR-2451 (P2) & $11,5^{*}$ & 12,9 \\
OR-1137 (M1) & 15,5 & 9,6 \\
OR-6252 (m1) & 14,1 & 9,0 \\
OR-6254 (m2) & &
\end{tabular}

*largo lingual/lingual length.

presenta, al igual que los ejemplares actuales de $P$. cancrivorus revisados. Se recomienda revisar este carácter en la propuesta de Baskin (2004).

\section{DISCUSIÓN}

El material descrito en este trabajo representa el primer reporte en el registro fósil de Nasua nasua y Procyon sp. para Venezuela y el norte de América del Sur. En este continente se ha reportado Nasua (probablemente N. nasua) para el Pleistoceno de Uruguay (Berta \& Marshall, 1978), Pleistoceno-Holoceno de Brasil (de Paula Couto, 1970) y el Holoceno tardío de Argentina (Ramírez et al., 2015), mientras que Procyon (P. cancrivorus) ha sido reportado para el Pleistoceno de Uruguay (Berta \& Marshall, 1978) y Argentina (Soibelzon et al., 2010) y Pleistoceno-Holoceno de Brasil (Paula Couto, 1970; Rodríguez et al., 2013). Debido a que estos prociónidos son de origen holártico (Koepfli et al., 2007), el material de El Breal de Orocual (ORS-16) representam los ejemplares de $N$. nasua y Procyon sp. más antiguo registrados hasta la fecha en América del Sur. La presencia de estos taxones para el Marplatense contradice los señalamientos de Simpson (1950), Soibelzon y Prevosti, (2012), Prevosti et al., (2013), Rodríguez et al. (2013), entre otros, en donde manifiestan que estos taxones no estaban presentes en las primeras etapas de la migración masiva entre las américas (GABI). Sin embargo, el material no resulta tan antiguo como para aceptar la hipótesis de Koepfli et al. (2007) en donde dice que Nasua y Procyon ingresaron a América del Sur contemporáneamente con Cyonasua, antes de la formación del corredor migratorio terrestre entre las américas $(<3,0-2,5 \mathrm{Ma}$.).

Es importante señalar que ambos géneros tienen una distribución amplia en América del Sur y parte de América 
Central, y están aún presentes en la región Neotropical (Linares, 1998; Sánchez \& Lew, 2012). En Venezuela, los representantes actuales de Nasua nasua se distribuyen en la región andina y al sur del río Orinoco, excluyendo de esta distribución a la biorregión de los llanos (Bisbal, 1989; Eisenberg, 1989; Linares, 1998; Prieto-Torres et al., 2015) (ver Figura 1). Las evidencias fósiles descritas en este trabajo nos permiten plantear el escenario donde esta especie probablemente sufrió una fragmentación y restricción de su hábitat en algún momento posterior al Pleistoceno temprano, probablemente debido a que las condiciones ambientales durante Plioceno tardío y Pleistoceno temprano fueron distintas a las del presente, favoreciendo la presencia en la región de este coatí.

Por otro lado, aunque el material de Procyon reportado en este trabajo presenta dudas con respecto a la asignación de especie, este género sigue estando presente en la actualidad en la región de Orocual (con reportes de $P$. cancrivorus; Linares, 1998). Koepfli et al. (2007) indican que la divergencia entre P. lotor y P. cancrivorus ocurrió en Norteamérica hace 5,0 a 5,7 Ma. Sin embargo, $P$. cancrivorus no está registrado en América del Sur sino hasta el Pleistoceno (Soibelzon et al., 2010).

Es probable que este ejemplar forme parte de una población que migró a América del Sur cercana a la divergencia de especies. Esto debido que, aunque existe alta variabilidad intragenérica e intraespecífica en este taxón, los caracteres como un hipocono en el M1 desplazado lingualmente (frecuentemente observado en material norteamericano de Procyon), las cúspides del trigónido del $\mathrm{m} 1$ diferenciadas como en $P$. lotor y un entoconúlido en la base del entocónido como en $P$. rexroandensis, señalan que el material de El Breal de Orocual tiene una morfología más afín con miembros de Norteamérica que con el miembro suramericano $P$. cancrivorus.

\section{CONCLUSIONES}

Los nuevos registros de Nasua y Procyon para el Marplatense de América del Sur indican que estos prociónidos migraron desde Norteamérica, en algún momento entre el Mioceno tardío y el Pleistoceno temprano. Esto pone en duda las hipótesis manejadas anteriormente sobre el ingreso de estos mamíferos al continente.

Dentro de los carnívoros de El Breal de Orocual (ORS16) se han descrito elementos únicos para América del Sur, como son los felinos Homotherium venezuelensis y Smilodon gracilis y un cánido del género Canis. Esta asociación guarda una relación más cercana en diversidad con asociaciones de Norteamérica. Esto complementa las observaciones realizadas en este trabajo sobre los elementos fósiles de Procyon sp., en donde el material de El Breal de Orocual se asemeja morfológicamente más con el miembro norteamericano $P$. lotor. Esto significa que esta región, al oriente de Venezuela, tiene evidencias de que el origen y evolución de los carnívoros en América del Sur fue más complejo de lo que se pensaba.

\section{AGRADECIMIENTOS}

Este trabajo está soportado por el proyecto 1096 del IVIC: Mapa Paleontológico de Venezuela. Queremos agradecer a Petróleos de Venezuela, S.A. (PDVSA), en especial a PDVSA Oriente, por la cooperación con este descubrimiento. Agradecemos el acceso al Museo de la Estación Biológica de Rancho Grande (MEBRG) y al apoyo de J. Sánchez, F. Bisbal y E.G. Carmargo. Un agradecimiento al X. Wang y al J. Dines del Natural History Museum of Los Angeles County por el acceso a la colección de Mamíferos. También queremos agradecer el apoyo dado por la T.Z. Fauverte y M. Morgado-Vargas en la Unidad de Microscopía Electrónica del Centro de Química-IVIC. Agradecemos al S. Moyà-Solà y R. Minwer-Barakat del Instituto Catalán de Paleontología (ICP), a S.Y. Onary-Alves de la Universidade de São Paulo (USP) y a los árbitros de la RBP (F. Prevosti y anónimo), por sus opiniones sobre el material fósil y sugerencias en el manuscrito.

\section{REFERENCIAS}

Ahrens, H.E. 2012. Craniodental characters and the relationships of Procyonidae (Mammalia: Carnivora). Zoological Journal of the Linnean Society, 164:669-713. doi:10.1111/j.10963642.2011.00778.x

Baskin, J.A. 1982. Tertiary Procyoninae (Mammalia: Carnivora) of North America. Journal of Vertebrate Paleontology, 2:71-93.

Baskin, J.A. 2004. Bassariscus and Probassariscus (Mammalia, Carnivora, Procyonidae) from the early Barstovian (middle Miocene). Journal of Vertebrate Paleontology, 24:709-720. doi:10.1671/0272-4634(2004)024[0709:BAPMCP]2.0.CO;2

Berta, A. \& Marshall, L.G. 1978. South America Carnivora, i-viii. In: F. Westphal (ed.) Fossilium catalogus, I. Animalia. The Hague (Dr. W. Junk), p. 1-48.

Carbón, J.; Schubert, C. \& Vaz, J.E.1992. Caracterización y edades termoluminiscentes de los sedimentos de la Formación Mesa, en dos localidades del sur de Anzoátegui (Venezuela). Acta Cientifica Venezolana, 43:387-391.

Croft, D.A. 2016. Horned armadillos and rafting monkeys: the fascinating fossil mammals of South America. Indiana University Press, 304 p.

Decker, D.M. 1991. Systematics of the coatis, genus Nasua (Mammalia: Procyonidae). Proceedings of the Biological Society of Washington, 104:370-386.

Forasiepi, A.M.; Soibelzon, L.H.; Gómez, C.S.; Sánchez, R.; Quiroz, L.I.; Jaramillo, C. \& Sánchez-Villagra, M.R. 2014. Carnivorans at the Great American Biotic Interchange: new discoveries from the northern neotropics. Naturwissenschaften, p. 1-10. doi:10.1007/s00114-014-1237-4.

Gidley, J.W. 1908. A fossil raccoon from a California Pleistocene cave deposit. Proceedings of the National Museum, 22:553-555.

Gilmore, L.S. 2013. Analysis of the Blancan Procyonids of Florida. Faculty of the Department of Geosciences, East Tennessee State University, Master of Science in Geosciences, $130 \mathrm{p}$.

Gompper, M.E. 1995. Nasua narica. Mammalian Species, 487:1-10.

Gompper, M.E. \& Decker, D.M. 1998. Nasua nasua. Mammalian Species, 580:1-9.

Koepfli, K.P.; Gompper, M.E.; Eizirik, E.; Ho, C.C.; Linden, L.; Maldonado, J.E. \& Wayne, R.K. 2007. Phylogeny of the Procyonidae (Mammalia: Carnivora): molecules, morphology 
and the great American interchange. Molecular Phylogenetics and Evolution, 43:1076-1095. doi:10.1016/j.ympev.2006.10.003

Linares, O.J. 1981. Tres nuevos carnívoros prociónidos fósiles del Mioceno de Norte y Sudamérica. Ameghiniana, 18:113-121.

Oelrich, T.M. 1953. Additional mammals from the Rexroad fauna. Journal of Mammalogy, 34:373-378. doi:10.2307/1375846

Patterson, B. \& Pascual, R. 1968. The fossil mammal fauna of South America. Quarterly Review of Biology, 43:409-451.

Paula-Couto, J.C. 1970. Paleontologia da região de Lagoa Santa, Minas Gerais, Brasil. Boletim Museo Historia Natural, 1:1-21.

Prevosti, F.J.; Forasiepi, A. \& Zimicz, N. 2013. The evolution of the Cenozoic terrestrial mammalian predator guild in South America: competition or replacement? Journal of Mammalian Evolution, 20:3-21. doi:10.1007/s10914-011-9175-9

Prevosti, F.J.; Soibelzon, L.H.; Patterson, B.D. \& Costa, L.P. 2012. Evolution of the South American carnivores (Mammalia, Carnivora): a paleontological perspective. In: D.B. Patterson \& L.P. Costa (eds.) Bones, clones, and Biomes: The History and Geography of Recent Neotropical Mammals, The University of Chicago Press, p. 102-122.

Ramírez, M.A.; Prevosti, F.J.; Acosta, A.; Buc, N. \& Loponte, D. 2015. On the presence of Nasua Storr (Carnivora, Procyonidae) in the Buenos Aires province in the late Holocene. Revista del Museo Argentino de Ciencias Naturales, 17:51-58.

Rincón, A.D.; Parra, G.; Prevosti, F.J.; Steadman, D. \& Alberdi, M.T. 2007. A new Pleistocene vertebrate assemblage in a tar pit from El Breal de Orocual, northeastern Venezuela. Journal of Vertebrate Paleontology, 27:134-135.

Rincón, A.D.; Parra, G.E.; Prevosti, F.J.; Alberdi, M.T. \& Bell, C.J. 2009. A preliminary assessment of the mammalian fauna from the Pliocene-Pleistocene El Breal de Orocual locality, Monagas State, Venezuela. Museum of Northern Arizona Bulletin, 65:593-620.

Rincón, A.D.; Prevosti, F.J. \& Parra, G.E. 2011. New saber-toothed cat records (Felidae: Machairodontinae) for the Pleistocene of Venezuela, and the Great American Biotic Interchange. Journal of Vertebrate Paleontology, 31:468-478. doi:10.1080/0272463 4.2011.550366
Rodríguez, S.G.; Soibelzon, L.H.; Rodrigues, S.; Morgan, C.C.; Bernardes, C.; Avilla, L. \& Lynch, E. 2013. First record of Procyon cancrivorus (G. Cuvier, 1798) (Carnivora, Procyonidae) in stratigraphic context in the Late Pleistocene of Brazil. Journal of South American Earth Sciences, 45:1-5. doi:org/10.1016/j. jsames.2013.01.004

Ruiz-Ramoni, D. 2016. Paleobiología y ecología evolutiva de los carnivoros fósiles (Eutheria: Mammalia) de los yacimientos de asfalto El Breal de Orocual y El Mene de Inciarte, en Venezuela. Postgrado en Ecología, Instituto Venezolano de Investigaciones Científicas, Tesis Doctoral, 218 p.

Ruiz-Ramoni, D.; Rincón, A.D.; Solórzano, A. \& Moyà-Solà, S. 2017. The first fossil Platyrrhini (Primates: Anthropoidea) from Venezuela: A capuchin monkey from the Plio-Pleistocene of El Breal de Orocual. Journal of Human Evolution, 105:127-131. doi:10.1016/j.jhevol.2017.01.012

Sánchez, H.J. \& Lew, D. 2012. Lista actualizada y comentada de los mamíferos de Venezuela. Memoria de la Fundación La Salle de Ciencias Naturales, 173-174:173-238..

Simpson, G.G. 1950. History of the fauna of Latin America. American Scientist, 38:361-389.

Soibelzon, L.H. \& Prevosti, F.J. 2007. Los carnívoros (Carnivora, Mammalia) terrestres del Cuaternario de América del Sur". In: G.X. Pons \& D. Vicens (eds.) Geomorfologia litoral i quaternari. Homenatge a Joan Cuerda Barceló, Palma de Mallorca, Societat d'Historia Natural de les Balears, p. 48-68 (Monografes 14).

Wolsan, M. \& Lange-Badré, B. 1996. An arctomorph carnivoran skull from the Phosphorites du Quercy and the origin of procyonids. Acta Palaeontologica Polonica, 41:277-298.

Woodburne, M.O. 2010. The Great American Biotic Interchange: dispersals, tectonics, climate, sea level and holding pens. Journal of Mammal Evolution, 17:245-264. doi:10.1007/s10914-0109144-8

Wrigth T. \& Lundelius, Jr. 1963. Post-Pleistocene raccoons from central Texas and their zoogeographic significance. Austin, Texas Memorial Museum, 21 p. (The Pearce Sellard Series 2).

Received in 25 January, 2017; Accepted in 30 December, 2017.

Apéndice 1. Ejemplares en colección utilizados para la comparación y análisis morfométricos.

Appendix 1. Collection specimens used for comparison and morphometric analysis.

\begin{tabular}{|c|c|c|}
\hline Procedencia & Número & Especie \\
\hline Baskin (1982) & & Arctonasua eurybates \\
\hline Baskin (1982) & & Arctonasua eurybates \\
\hline Baskin (1982) & & Arctonasua floridana \\
\hline Baskin (1982) & & Arctonasua floridana \\
\hline Baskin (1982) & & Arctonasua gracilis \\
\hline Baskin (1982) & & Arctonasua minima \\
\hline Venezuela-Bolívar & EBRG 602 & Bassaricyon beddardi \\
\hline Venezuela-Bolívar & EBRG 603 & Bassaricyon beddardi \\
\hline Venezuela-Bolívar & EBRG 1628 & Bassaricyon beddardi \\
\hline Venezuela-Bolívar & EBRG 1629 & Bassaricyon beddardi \\
\hline Venezuela-Bolívar & EBRG 1630 & Bassaricyon beddardi \\
\hline Venezuela-Bolívar & EBRG 1631 & Bassaricyon beddardi \\
\hline Forasiepi et al. (2014) & MACN 7955 & Cyonasua clausa \\
\hline Forasiepi et al. (2014) & MMMP 645 S & Cyonasua groeberi \\
\hline Forasiepi et al. (2014) & MLP 34-VI-20-6 & Cyonasua lutaria \\
\hline Forasiepi et al. (2014) & MMP 1005 M & Cyonasua lutaria \\
\hline Linares (1981) & AM 45985 & Cyonasua pascuali \\
\hline México-Colima & LAM 59443 & Nasua narica \\
\hline México-Colima & LAM 59442 & Nasua narica \\
\hline México-Colima & LAM 29312 & Nasua narica \\
\hline México-Colima & LAM 59446 & Nasua narica \\
\hline
\end{tabular}

\begin{tabular}{lcc}
\hline \multicolumn{1}{c}{ Procedencia } & Número & Especie \\
\hline Venezuela-Aragua & EBRG 1090 & Nasua nasua \\
Venezuela-Bolívar & EBRG 1623 & Nasua nasua \\
Venezuela-Sucre & EBRG 1646 & Nasua nasua \\
Venezuela-Aragua & EBRG 1706 & Nasua nasua \\
Venezuela-Aragua & EBRG 2056 & Nasuella meridensis \\
Venezuela-Cojedes & EBRG 24244 & Nasuella meridensis \\
Venezuela-Carabobo & EBRG 4 & Procyon cancrivorus \\
Venezuela-Guárico & EBRG 404 & Procyon cancrivorus \\
Venezuela-Bolívar & EBRG 767 & Procyon cancrivorus \\
Venezuela-Guárico & EBRG 799 & Procyon cancrivorus \\
Venezuela-Guárico & EBRG 800 & Procyon cancrivorus \\
Wrigth y Lundelius Jr (1963) & & Procyon lotor \\
Wrigth y Lundelius Jr (1963) & & Procyon lotor \\
USA-Arizona & LAM 8368 & Procyon lotor \\
Estados Unidos-California & LAM 7240 & Procyon lotor \\
Estados-Arizona & LAM 458 & Procyon lotor \\
Estados Unidos-California & LAM 7241 & Procyon lotor \\
Estados Unidos-California & LAM 52261 & Procyon lotor \\
Estados Unidos-California & LAM 31579 & Procyon lotor \\
Linares (1981) & F: AM 25210 & Protoprocyon savagei \\
\hline
\end{tabular}

